\title{
Transcriptome analysis identifies DMRT3 in nasal polyp epithelial cells of patients suffering from Nonsteroidal anti-inflammatory drugs- Exacerbated Respiratory Disease (N-ERD)
}

\author{
VS Priyadharshini ${ }^{1}$, Alejandro Jiménez-Chobillon ${ }^{1}$, Jos de Graaf ${ }^{2}$, Raul Gutiérrez de \\ Velasco $^{1}$, Christina Gratziou ${ }^{3}$, Fernando Ramirez-Jimenez ${ }^{1}$, and Luis Teran ${ }^{1}$ \\ ${ }^{1}$ Instituto Nacional de Enfermedades Respiratorias \\ ${ }^{2}$ Translationale Onkologie an der Universitätsmedizin der Johannes Gutenberg-Universität \\ Mainz gGmbH (TRON) \\ ${ }^{3}$ Evgenidio Hospital
}

October 6, 2020

\begin{abstract}
Background: N-ERD is a syndrome characterized by chronic rhinosinusitis nasal polypsasthma and aspirin intolerance. An imbalance of eicosanoid metabolism with overproduction of CysLTs has been associated with N-ERD however, the precise mechanisms underlying N-ERD are unknown. Objective: To establish the transcriptome of the nasal polyp airway epithelial cells derived from N-ERD patients to uncover the gene expression patterns during this disease. Methods: Nasal airway epithelial cells were isolated from $12 \mathrm{~N}$-ERD polyps and $9 \mathrm{~N}$-ERD non polyp nasal mucosa as controls from the same subjects. RNA was sequenced on the Illumina HiSeq 2500. Potential gene candidate DMRT3 was selected from the differentially expressed genes for validation. Results: Comparative transcriptome profiling of nasal epithelial cells was achieved in N-ERD. 18 genes had twofold mean regulation expression differences or greater. 5 genes were upregulated including DMRT3 and 12 genes were down-regulated. Differentially regulated genes included inflammation, defense and immunity. Significantly enriched pathways were metabolic process and embryonic development. ELISA results of DMRT3 in N-ERD patients was significantly upregulated when compared to controls $(\mathrm{p}=0.03)$. IHC of N-ERD nasal polyps localised DMRT3 and was predominantly released in the airway epithelia. These results corroborate with our findings. Conclusion: Findings suggest that DMRT3 could be potentially involved in nasal polyps development in N-ERD patients. Known functions of DMRT3 include nucleic acid binding and highly expressed during embryonic development. Several genes are downregulated, hinting dedifferentiation phenomenon in N-ERD polyps. However, further studies are required to confirm the exact mechanism of polyps formation in N-ERD patients.
\end{abstract}

\section{Introduction}

Nonsteroidal anti-inflammatory drugs -exacerbated respiratory disease (N-ERD) is a syndrome characterized by rhinosinusitis nasal polyps asthma and aspirin intolerance. N-ERD is also known as Aspirin Exacerbated Respiratory Disease (AERD), aspirin sensitive asthma or aspirin intolerant asthma.Up to $73 \%$ of N-ERD patients develop atopy although specific IgE antibodies to aspirin have not been identified. The pathogenic mechanisms associated with N-ERD include overproduction of cysteinyl leukotrienes (CysLTs), increased CysLTR1 expression in the airway mucosa and decreased lipoxin and PGE2 synthesis. ${ }^{1}$ Anti-leukotriene therapy ameliorates asthma symptoms in aspirin-intolerant patients. However, nasal polyps development remains a significant challenge in N-ERD management. Nasal polyps are inflammatory pseudotumoral masses that most frequently start to grow from the ostiomeatal complex and the cells of the anterior ethmoidal sinus. They can affect the totality of the remaining sinus cavities including the posterior ethmoidal cells, the maxillary, and the frontal or the sphenoidal sinuses, and they also can extend to the olfactory cleft, the 
sphenoethmoidal recess, and the nasal cavities. ${ }^{2}$ Nasal polyposis in N-ERD patients is present in up to 80 to $90 \%$ of patients and tends to be more aggressive and difficult to treat medically, also presenting with higher recurrence rates after surgery. A survey in 190 N-ERD patients suffering NPs that analyzed perceptions and quality of life showed that chronic nasal symptoms followed by decreased sense of smell were reported to have the most significant impact on quality of life - (40\% approximately) and patients who lost their smell $(34 \%)$ reported that they missed the enjoyment of food and eating the most. ${ }^{3}$ On the other hand, the surgical removal of nasal polyps has been shown to decrease both the urinary LTE4 levels and asthma exacerbations. $^{4-8}$

The mucosal lining of the nasal polyps is a columnar glandular pseudostratified epithelium that also plays a significant role in cytokine and inflammatory mediator release and it has been implicated in nasal polyps in N-ERD. A study conducted by Picado et al. showed that COX-2 was downregulated in NP epithelial cells (ECs) derived from aspirin sensitive patients and proposed that dysregulation of COX-2 could play an important role in nasal polyps. ${ }^{9}$ Subsequently, Kowalski et al. found NP ECs from N-ERD patients generated 3 fold less prostaglandin E2(PGE2), compared with aspirin tolerant subject. Prostaglandin E2 (PGE2) is known to have bronchodilator properties and decreased production of this mediator could account to N-ERD development. ${ }^{10}$ In 2007 , the same group also investigated the ability of nasal polyps epithelial cells to produce higher levels of stem cell factor when compared with aspirin tolerant patients: they proposed that increased expression and secretion of stem cell factor, a chemotactic growth and differentiation factor for mast cells, accounted for increased mast cell infiltration and activation ${ }^{11}$ in N-ERD. In this study, we have investigated N-ERD polyps epithelial cell transcriptome.

\section{Methods}

\section{Study Design}

Subjects were divided into three groups. Group A subjects $(\mathrm{n}=12)$ included N-ERD patients who underwent routine polypectomy for a therapeutic reason: two types of samples were collected from these patients; nasal polyps which were removed during polypectomy $(\mathrm{n}=12)$; and nasal tissue from the middle turbinate which was collected from the non-polyp nasal mucosa $(n=8)$. For validation studies, Nasal lavages were performed in these two groups of patients. Group B, N-ERD patients $(\mathrm{n}=12)$ and Group $\mathrm{C}$, non-atopic, healthy controls $(\mathrm{n}=8)$, were included.

\section{Subjects}

Thirty-two subjects participated in this study (table 1). Their atopic status was investigated by skin-prick testing with different allergens. N-ERD was defined as the presence of asthma, nasal polyps or previous polyp surgery, NSAID intolerance (nasal challenge with lysin-aspirin or two severe reactions to NSAID previously). Asthma was established as typical persistent symptoms: (shortness of breath, wheezing, chest tightness, and cough); plus $>12 \%$ or $200 \mathrm{ml}$ increase of forced expiratory volume in 1 second (FEV1); post-bronchodilatator spirometry (Mater Screen, Jaegger-Germany). Allergy sensitization was evaluated with skin prick test with a kit of 40 allergens (Alk-abello; Massachusetts, USA), and levels of total IgE (Architect i2000, Roche, Germany) and eosinophils counts were measured in blood (Beckman coulter LH750, USA). N-ERD subjects were given dexamethasone before surgery. Patients who volunteered for nasal lavage collection were asked to stop the use of inhaled corticosteroid for 7 days before sample collection. However, bronchodilators and inhaled corticosteroids were not withheld. The study was approved by the Bioethics and Science Committee in Research, with protocol number B02-14 and Institutional Review Board at National Institute of Respiratory Diseases (INER) Ismael Cosio Villegas.

\section{Cell Culture}

Nasal airway epithelial cells were isolated from nasal polyps and N-ERD non-affected nasal mucosal epithelial cells, which were obtained from N-ERD patients undergoing polypectomy for therapeutic reasons. Cells were grown in Bronchial Epithelial Cell Growth Medium BulletKit (BEGM), Clonetics, USA under standard conditions. When cells reached $80 \%$ confluence, they were harvested and the pellet was dissolved in 0.01 
M PBS, and 3 to 4 volumes of RNAlater (Invitrogen, USA) was added for future RNA extraction and was stored in $-70^{\circ} \mathrm{C}$.

\section{RNA extraction and quantification}

RNA extraction was performed using the Qiagen RNeasy micro kit using standard protocol. RNA and cDNA quantifications were done using Qubit 3.0 fluorometer, (Invitrogen) using the manufacturer's instructions. With regard to quality assurance, the RNA Integrity Number (RIN) of RNA obtained from patient samples was determined using microfluidics analysis on the Agilent bioanalyzer Pico RNA and HS DNA kits. Only samples with a RIN greater than 7 were subjected to RNA-seq.

\section{RNA sequencing and Data Analysis}

Library preparation was performed using Illumina Truseq RNA V2 kit according to the manufacturer's protocol, libraries were clustered using TruSeq PE Cluster Kit v3-cBot-HS and RNA Seq was performed on Illumina's HiSeq2500, generating paired end 2 x 50 nucleotide reads using TruSeq SBS Kit v3 - HS. The read mapping to specific regions of the reference genome was done with hisat2. Counting was done with a flexible overlap approach. Up-regulated genes should have a logarithmic fold change $>\log 2$ (1.5) and down-regulated genes $<-\log 2$ (1.5). The p-values were adjusted stepwise using the Benjamini-Hochberg procedure, p-value at or below 0.05. Differentially expressed genes are displayed in a heatmap. R package DESeq2 was used for differential expression analysis. RNA sequencing was carried out at Translational Oncology, University Medical Center of the Johannes Gutenberg University; Mainz. Gene expression data has been deposited in NCBI’s Gene Expresssion Omnibus (GEO), Accession number GSE158277.

\section{Immunohistochemistry}

Nasal polyps tissues were fixed in paraformaldehyde and paraffin-embedded. Immunohistochemistry for DMRT3 was performed using vectastain ABC staining (Vector Laboratories Inc., USA), DMRT3 antibody was purchased from Thermofisher Scientific and diluted as described by the manufacturer. The sections were counterstained using Mayer's haematoxylin (Vector Laboratories Inc., USA).

\section{Nasal lavage}

Nasal lavages were performed by instilling $10 \mathrm{ml}$ of sterile physiological saline solution into each nostril; the fluid was expelled after 10s. About $8 \mathrm{ml}$ of the nasal secretion was collected routinely from each patient (the extent of polyps did not modify the procedure or the collected volume). Nasal lavage samples were homogenized by vigorous shaking and centrifuged at $3000 \mathrm{rpm}$ for $10 \mathrm{~min}$ and then maintained at $20^{\circ} \mathrm{C}$ until their use. Before ELISA measurements, nasal lavages were concentrated 10 times using Amiconß Ultra (Millipore, Billerica, MA, USA) according to manufacturer's protocol.

\section{DMRT3 ELISA measurements}

Measurements of DMRT3 was performed in 8 times concentrated nasal lavage fluid using a two-antibody sandwich ELISA (MyBioSource) as previously described. ${ }^{12-13}$ The concentration of DMRT3 in samples was calculated from the standard curve. The sensitivity of this kit is $10 \mathrm{pg} / \mathrm{ml}$. The detection range of this kit is $62.5 \mathrm{pg} / \mathrm{ml}-2000 \mathrm{pg} / \mathrm{ml}$.

\section{Statistical analysis}

Descriptive statistics were expressed as median (interquartile range); the nonparametric Mann-Whitney test was used for comparison of concentrations between the groups and graphs were calculated using STATA statistical software, version 13 (Stata Corp LP, College Station, Texas). $\mathrm{p}<0.05$ was considered statistically significant.

\section{Results}

\section{Characteristics of participants}


In this study, we have investigated the transcriptome of nasal epithelial cells derived from 12 patients suffering from N-ERD (NPEC) compared with nasal epithelial cells from the non-affected nasal area from the same patients (NNAEC) in order to reduce genetic heterogeneity (N-ERD patients served as their own control). Nasal epithelial cells were successfully isolated from all subjects with exception of three control nasal mucosal tissues (nasal tissue was not adequate for cell culture from two subjects and in one of them, it was not collected because of disease severity). All patients were given $8 \mathrm{mg}$ dexamethasone prior to the nasal polypectomy. For validation experiments, two new group of subjects participated in the study, including the second group of N-ERD patients suffering nasal polyps $(n=12)$ and a healthy control group $(n=8)$ who underwent nasal lavage. None of the subjects in group B and C was taking oral steroids. N-ERD patients stopped inhaled steroids 7 days before the nasal lavage to prove corticosteroids did not affect mediator measurements. Clinical characteristics of subjects are shown in table 1.

\section{Differential gene expression analysis}

Airway epithelial cells derived from both N-ERD Polyp and NNAEC mucosa were successfully sequenced. A total of 364 genes were differentially expressed in NPEC versus NNAEC (figure 1). However, only 18 genes achieved statistical significance after Benjamini-Hochberg adjustment for false discovery rate: 6 genes were upregulated and 12 genes were downregulated (table 2). The full list of genes is provided in the supplementary material. Functional analysis of these genes using GO revealed categories that are associated with retinol metabolism, steroid hormone biosynthesis, primary bile acid biosynthesis, phenylalanine metabolism (table 3), plasma membrane organization, macroautophagy, leukotriene metabolic process, protein localization to the membrane, drug catabolic processes, and neuron cell-cell adhesion. Pathview toolset (Bioconductor) was used for pathway-based data integration and visualization (table 4). The data discussed in this publication have been deposited in NCBI's Gene Expression Omnibus ${ }^{14}$ and are accessible through GEO Series accession number GSE158277

\section{Measurements of DMRT3}

DMRT3 is involved in embryonic development, which may be associated with nasal polyp formation. Thus, we have investigated whether DMRT3 protein is present in the nasal lavage of patients suffering from N-ERD using a specific ELISA. At this time, a group of normal healthy subjects participated as control. Interestingly, levels of DMRT3 were significantly elevated in nasal lavage fluids obtained from N-ERD patients compared with normal controls $(457 \mathrm{pg} / \mathrm{ml}(363-942 \mathrm{pg} / \mathrm{ml})$ versus $275 \mathrm{pg} / \mathrm{ml}(275-610 \mathrm{pg} / \mathrm{ml})$. (Figure 2). This finding is in agreement with the high differential gene expression observed in the RNA-Seq experiment.

\section{DMRT3 immunoreactivity in nasal polyps}

To investigate DMRT3 immunoreactivity in nasal polyps we applied immunohistochemistry to nasal polyp biopsies derived from N-ERD patients. DMRT3 immunoreactivity was localized predominantly to the airway epithelium (Figure 3) and there was sparse immunoreactivity localized to mononuclear cells in the subepithelial tissue.

\section{Discussion}

Nasal polyp management remains a significant challenge in N-ERD. In the present study, we investigated the transcriptome of nasal airway epithelial cells and identified several differentially regulated genes associated with retinol metabolism, steroid hormone biosynthesis, primary bile acid biosynthesis, phenylalanine metabolism. Of particular interest was the identification of DMRT3, which is a gene involved in embryonic development. Interestingly, by ELISA, we demonstrated that DMRT3 is released in increased concentration in the upper airways of N-ERD patients suggesting it is involved in the pathogenesis of this disease. To our knowledge, this is the first study to utilize RNA-Seq gene expression profiling of N-ERD polyps derived epithelial cells.

Medical treatment of nasal polyps in N-ERD patients includes topical- and oral corticosteroids, antibiotics and surgical intervention. However, up to $40 \%$ of NPs patients show recurrence and require an additional endoscopic sinus surgery within 18 months. ${ }^{15}$ Clinical trials with novel therapeutic biologics have recently 
tested anti-IL-5, anti-IL-4, anti-IL-13 and anti-IgE. However, most of these treatments have limited impact on nasal polyps. ${ }^{16-18}$ Using RNA sequencing, Peng et al. showed defective host defenses and heightened inflammation response in whole tissue nasal polyps. ${ }^{19}$

To investigate local transcriptomic changes involved in nasal polyp pathogenesis, we performed sequencing of a nasal polyp- and healthy nasal epithelial cells from N-ERD patients that revealed 18 differentially regulated genes. Of particular interest was the identification of the DMRT3 gene, which was highly overexpressed in NPECs. This gene is a member of the DMRT family, known to play a conserved role in sex determination, sexual dimorphism and other aspects of sexual reproduction. ${ }^{20-21}$ DMRT1 is the predominant gene involved in testis development; however, weak DMRT3 expression has been reported in the developing testis of mice male embryos (but not in ovaries of females). ${ }^{22}$ This finding contrasts with the DMRT1 expression profile in chicken embryos, in which DMRT3 transcripts were detectable in the Müllerian ducts but not in the gonads. Thus, it seems that there is some interspecies variation on DMRT3 regulation. Li et al. showed a restricted expression pattern of DMRT3 in zebrafish neural tube and olfactory placode during embryogenesis, and in developing germ cells of both undifferentiated and adult gonads. ${ }^{23}$ In the juvenile zebrafish, DMRT3 gene expression is first detected in undifferentiated gonads on day 17 post-fertilization while In adults, DMRT3 is expressed only in the developing germ cells of both gonads, specifically in spermatogonia, spermatocytes and developing oocytes. These results suggest that as a DNA-binding protein, zebrafish DMRT3 may function as a transcription factor to exert potential roles in the development of the olfactory placode, the neural tube and germ cells. In humans, DMRT1, DMRT3 and DMRT2 (9p24.3) are associated with human testicular dysgenesis and XY male-to-female sex reversal. ${ }^{24-25}$ DM domain proteins might also play a role in human cancer: amplification and overexpression of DMRT1 are associated with spermatocytic seminoma. ${ }^{26}$ At the time of writing the manuscript, DMRT3 was found to be overexpressed in patients suffering from colorectal cancer, and it was associated with the expression of SND1 rs118049207. ${ }^{27}$ Stratification analyses in this last report showed that SND1rs118049207 multiplicatively interacted with the sex and drinking status of the patients to increase their colorectal cancer risk. Given its participation in embryogenesis it is tempting to hypothesize that DMRT3 is involved in nasal polyp formation. It has been shown that the surgical removal of nasal polyps improves asthma symptoms and a decreases urinary LTE4 levels. Thus, the development of new drugs which neutralize this gene may not only antagonize nasal polyp formation but it may also reduce

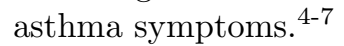

To investigate whether DMRT3 is released into airway epithelial lining fluid, we have measured levels of this protein in nasal fluid. The epithelial lining fluid (ELF) forms a thin fluid layer that covers the nasal mucosa and reflects the ongoing changes taking place in some of the pathological processes related to the progression of disorders in the upper airways. At this time, measurements were performed in the second group of N-ERD patients and comparisons were made with normal subjects. In contrast to N-ERD patients who underwent nasal polyp removal, this new group of N-ERD subjects did not receive oral steroids which excludes the possibility of gene expression alteration due to steroids. Interestingly, DMRT3 levels were increased in the nasal lavages of N-ERD patients as compared with healthy subjects which suggest this protein is released in the upper airways. Moreover, by immunohistochemistry, we further demonstrated that DMRT3 immunoreactivity is predominantly localized to the bronchial epithelium, further supporting its synthesis by this cell type. To our knowledge, this is the first report showing DMRT3 is produced in the upper airways in humans.

In the present study, several additional genes were found to be differentially regulated, and the number of genes down-regulated were higher than upregulated in N-ERD polyps, hinting at dedifferentiation ${ }^{28}$ and transdifferentiation ${ }^{29}$ phenomena. For example, NTSR1 inhibition induces intrinsic apoptosis via downregulation of Bcl-w and Bcl-2 in glioblastoma cells. ${ }^{30}$ Kontovounisios et al. showed that neurotensin levels were lower in adenomatous polyps as compared with adenocarcinoma. ${ }^{31}$ On the other hand, Brun $\mathrm{P}$ et al. showed that neurotensin (NT) significantly increased COX-2 mRNA levels by 2.4-fold and stimulated PGE2 release in HT-29 cells. Neurotensin and NTR1 are part of the network activated after mucosal injuries and NT stimulates epithelial restitution at least, in part, through a COX-2 dependent pathway. ${ }^{32}$ Interestingly, NTSR1 was significantly downregulated in N-ERD polyps when compared to non-polyp mucosa, and retinoic acid 
metabolism pathway was significantly enriched in our results, suggesting downregulation of NTSR1 might influence retinol metabolism in N-ERD polyps. ${ }^{33}$ Retinoids are essential for the maintenance of epithelial differentiation. As such, they play a fundamental role in chemoprevention of epithelial carcinogenesis and in differentiation therapy. ${ }^{34}$ Degradation of retinol in N-ERD polyps suggests a dedifferentiation phenomenon. AEBP1 is an ECM-associated protein with crucial functions in both embryonic development and adult tissue repair. ${ }^{35}$ Similarly, LAPTM5 was downregulated in our findings, implying its potential role in polyp formation.Nuylan M et al. in 2016 reported that LAPTM5 (Human Retinoic Acid-Inducible E3 Protein) mRNA levels where frequently decreased in various cancer cell lines, and its low expression in patients with esophageal squamous cell carcinoma (ESCC) and non-small cell lung cancer(NSCLC) was significantly correlated with substandard prognosis. ${ }^{36}$ Conversely, CPXM2 was up-regulated; Niu G et al. reported CPXM2 upregulation is promoting gastric cancer aggressiveness via epithelial to mesenchymal transition (EMT) modulation. ${ }^{37}$ Future studies should further decipher the role of these genes in N-ERD.

To date this is the first study to analyze the transcriptome of airway epithelial cells derived from a nasal polyp- and non-nasal polyp tissue derived from same patients. Strengths of having analyzed matched samples include a) isolating NAECs from the same subjects provide a good control for individual differences and, as a result, prevents random error. Most previous studies have used nasal tissue. For example, Stevens WW et al present a general analysis of gene expression levels using scRNA-seq comparing NP of AERD and CRSwNP patients. ALOX15 was predominantly expressed by apical epithelial cells and significantly increased in AERD compared to CRSwNP NP. ${ }^{38}$ They reported that downstream mediators in the 15-LO pathway, including 15-HETE and 15-oxo- ETE, may be important factors contributing to AERD pathophysiology. they also discovered the presence of a trans-metabolic process how epithelial cells convert AA to 15-HETE (by 15-LO) and nearby mast cells convert 15-HETE to 15-oxo-ETE (by HPGD). b) genetic variability is reduced and c) a large sample size is not required as the sampling is done repeatedly from the nasal passage of the same individual. Future studies should investigate the role of DMRT3 in other nasal polyp related diseases.

In summary, the present study has revealed 18 differentially expressed genes in NPEC using RNA sequencing. Of particular interest was the identification of DMRT3, which is a gene involved in embryonic development. ELISA measurements showed DMRT3 is released in high concentrations in the nasal airway epithelial fluid of N-ERD patients. This study further confirms the value of performing a matched-case control approach as a valuable tool to uncover mechanism involved in N-ERD polyps, and it demonstrates that careful study design allowed uncovering local gene activation. All together suggests that DMRT3 may become a potential molecular target for therapeutic intervention in N-ERD.

\section{Disclosure statement:}

All the authors declarethere is nothing to disclose.

\section{Conflicts of interest:}

All the authors declare no competing interests in association with the present work.

\section{Acknowledgements:}

Authors like to thank our lab technician Mr. Felipe Fuentes Arenas for his help in transporting samples to the laboratory.

\section{Ethics on Human Research}

This investigation was carry out according to articles $96^{\circ}$ and $98^{\circ}$, the fifth title of the General Mexican Health Law. Human research is based on ethical principles and participants have signed an informed consent letter. The protocols will be performed by health professionals. (Article 100 of the General Health Law). This project has the approval of the INER ethics committee, project B02-14.

\section{Support statement:}

Funded by the Consejo Nacional de Ciencia y Tecnología (CONACYT) - Project code-272892. 


\section{Abbreviations}

ACLP / AEBP1- Adipocyte Enhancer Binding Protein 1

Bcl- B-cell lymphoma 2

BEGM- Bronchial Epithelial Cell Growth Medium

cDNA- Complementary DNA

CNS- Central Nervous System

COX- Cyclooxygenase

EC- Epithelial cell

CysLT- Cysteinyl leukotrienes

DMRT3- Doublesex and mab-3 related transcription factor 3

DNA- Deoxyribonucleic acid

ECM- Extra Cellular Matrix

ELF- Epithelial Lining Fluid

ELISA- Enzyme linked immune sorbent assay

EMT- Epithelial-mesenchymal transition

ESCC- esophageal squamous cell carcinoma

FEV1- Forced expiratory volume in 1 second

GO- Gene Ontology

HT- High-Throughput

Ig E- Immunoglobulin E

IL- Interleukins

LAPTM5- Lysosomal Protein Transmembrane 5

N-ERD- NSAID-exacerbated respiratory disease

NSAID- Nonsteroidal anti-inflammatory drugs

NP- Nasal Polyps

NPEC -Nasal polyp epithelial cell

NNAEC -Non affected nasal area epithelial cell

NSCLC- Non small cell lung cancer

NTR1/NTSR1- Neurotensin receptor 1

PBS- Phosphate-buffered saline

PGE2- Prostaglandin E2

\section{References}

1. Rodriguez-Jimenez JC, Moreno-Paz FJ, Teran LM, Guani-Guerra E. Aspirin exacerbated respiratory disease: Current topics and trends. Respir Med. 2018 Feb;135:62-75. doi: 10.1016/j.rmed.2018.01.002. Epub 2018 Jan 10. PMID: 29414455. 
2. Lezcano-Meza D, Davila-Davila B, Vega-Miranda A, Negrete-Garcia MC, Teran LM. Interleukin (IL)-4 and to a lesser extent either IL-13 or interferon-gamma regulate the production of eotaxin-2/CCL24 in nasal polyps. Allergy. 2003;58(10):1011-1017.

3. Ta V, White AA. Survey-Defined Patient Experiences With Aspirin-Exacerbated Respiratory Disease. J Allergy ClinImmunolPract. 2015 Sep-Oct;3(5):711-8. doi: 10.1016/j.jaip.2015.03.001. Epub 2015 Apr 7. PMID: 25858054.

4. Taniguchi M, Mitsui C, Hayashi $\mathrm{H}$, et al. Aspirin-exacerbated respiratory disease (AERD): Current understanding of AERD. Allergol Int. 2019;68(3):289-295. doi: 10.1016/j.alit.2019.05.001

5. Langdon C, Mullol J. Nasal polyps in patients with asthma: prevalence, impact, and management challenges. J Asthma Allergy. 2016; 9:45-53. Published 2016 Mar 14. doi:10.2147/JAA.S86251

6. DeMarcantonio MA, Han JK. Nasal polyps: pathogenesis and treatment implications. OtolaryngolClin North Am. 2011;44(3):685-ix. doi: 10.1016/j.otc.2011.03.005

7. Ebbens FA, Toppila-Salmi SK, Renkonen JA, et al. Endothelial L-selectin ligand expression in nasal polyps. Allergy. 2010;65(1):95-102. doi:10.1111/j.1398-9995.2009. 01986.x

8. Lezcano-Meza D, Negrete-Garcia MC, Dante-Escobedo M, Teran LM. The monocyte-derived chemokine is released in the bronchoalveolar lavage fluid of steady-state asthmatics. Allergy. 2003;58(11):1125-1130. doi:10.1034/j.1398-9995.2003.00273.x

9. Picado C, Fernandez-Morata JC, Juan M, et al. Cyclooxygenase-2 mRNA is downexpressed in nasal polyps from aspirin-sensitive asthmatics. Am J RespirCrit Care Med. 1999;160(1):291-296. doi:10.1164/ajrccm.160.1.9808048

10. Kowalski ML, Pawliczak R, Wozniak J, Siuda K, Poniatowska M, Iwaszkiewicz J, Kornatowski T, Kaliner MA. Differential metabolism of arachidonic acid in nasal polyp epithelial cells cultured from aspirin-sensitive and aspirin-tolerant patients. Am J RespirCrit Care Med. 2000 Feb;161(2 Pt 1):391-8. doi: 10.1164/ajrccm.161.2.9902034. PMID: 10673176.

11. Santiago J, Hernandez-Cruz JL, Manjarrez-Zavala ME, Montes-Vizuet R, Rosete-Olvera DP, TapiaDiaz AM, Zepeda-Peney H, Teran LM. Role of monocyte chemotactic protein-3 and -4 in children with virus exacerbation of asthma. Eur Respir J. 2008 Nov;32(5):1243-9. doi: 10.1183/09031936.00085107. Epub 2008 Jun 25. PMID: 18579545.

12. Montes-Vizuet R, Vega-Miranda A, Valencia-Maqueda E, Negrete-Garcia MC, Velasquez JR, Teran LM. CC chemokine ligand 1 is released into the airways of atopic asthmatics. Eur Respir J. 2006 Jul;28(1):59-67. doi: 10.1183/09031936.06.00134304. Epub 2006 Mar 15. PMID: 16540498.

13. Lezcano-Meza D, Negrete-Garcia MC, Dante-Escobedo M, Teran LM. The monocyte-derived chemokine is released in the bronchoalveolar lavage fluid of steady-state asthmatics. Allergy. 2003;58(11):1125-1130. doi:10.1034/j.1398-9995.2003.00273.x

14. Edgar R, Domrachev M, Lash AE. Gene Expression Omnibus: NCBI gene expression and hybridization array data repository. Nucleic Acids Res. 2002 Jan 1;30(1):207-10. doi: 10.1093/nar/30.1.207. PMID: 11752295; PMCID: PMC99122.

15. DeConde AS, Mace JC, Levy JM, Rudmik L, Alt JA, Smith TL. Prevalence of polyp recurrence after endoscopic sinus surgery for chronic rhinosinusitis with nasal polyposis. Laryngoscope. 2017 Mar;127(3):550-555. doi: 10.1002/lary.26391. Epub 2016 Nov 12. PMID: 27859303; PMCID: PMC5321782.

16. Gevaert P, Van Bruaene N, Cattaert T, Van Steen K, Van Zele T, Acke F, De Ruyck N, Blomme K, Sousa AR, Marshall RP, Bachert C. Mepolizumab, a humanized anti-IL-5 mAb, as a treatment option for severe nasal polyposis. J Allergy Clin Immunol. 2011 Nov; 128(5):989-95.e1-8. doi: 10.1016/j.jaci.2011.07.056. Epub 2011 Sep 28. PMID: 21958585.

17. Gevaert P, Calus L, Van Zele T, Blomme K, De Ruyck N, Bauters W, Hellings P, Brusselle G, De Bacquer D, van Cauwenberge P, Bachert C. Omalizumab is effective in allergic and nonallergic patients with nasal polyps and asthma. J Allergy Clin Immunol. 2013 Jan;131(1):110-6.e1. doi: 10.1016/j.jaci.2012.07.047. Epub 2012 Sep 27. PMID: 23021878.

18. Bachert C, Mannent L, Naclerio RM, et al. Effect of Subcutaneous Dupilumab on Nasal Polyp Burden in Patients With Chronic Sinusitis and Nasal Polyposis: A Randomized Clinical Trial. JAMA. 
2016;315(5):469-479. doi:10.1001/jama.2015.19330

19. Peng Y, Zi XX, Tian TF, et al. Whole-transcriptome sequencing reveals heightened inflammation and defective host defence responses in chronic rhinosinusitis with nasal polyps. Eur Respir J. 2019;54(5):1900732. Published 2019 Nov 14. doi:10.1183/13993003.00732-2019

20. Bellefroid EJ, Leclere L, Saulnier A, Keruzore M, Sirakov M, Vervoort M, DeClercq S. Expanding roles for the evolutionarily conserved Dmrt sex transcriptional regulators during embryogenesis. Cell Mol Life Sci. 2013 Oct;70(20):3829-45. doi: 10.1007/s00018-013-1288-2.

21. Kopp A (2012) Dmrt genes in the development and evolution of sexual dimorphism. Trends Genet $28(4): 175-184$.

22. Matson CK, Zarkower D (2012) Sex and the singular DM domain: insights into sexual regulation, evolution and plasticity. Nat Rev Genet 13(3):163-1745.

23. Li Q, Zhou X, Guo Y, Shang X, Chen H, Lu H, Cheng H, Zhou R. Nuclear localization, DNA binding and restricted expression in neural and germ cells of zebrafish Dmrt3. Biol Cell. 2008 Aug;100(8):45363. doi: 10.1042/BC20070114. PMID: 18282142.

24. Smith CA, Hurley TM, McClive PJ, Sinclair AH (2002) Restricted expression of DMRT3 in chicken and mouse embryos.Gene Expr Patterns 2(1-2):69-72.

25. Raymond CS, Parker ED, Kettlewell JR, Brown LG, Page DC, Kusz K, Jaruzelska J, Reinberg Y, Flejter WL, Bardwell VJ, Hirsch B, Zarkower D: A region of human chromosome 9p required for testis development contains two genes related to known sexual regulators. Hum Mol Genet 1999, 8(6):989-996.

26. Murphy MW, Zarkower D, Bardwell VJ. Vertebrate DM domain proteins bind similar DNA sequences and can heterodimerize on DNA. BMC Mol Biol. 2007 Jul 2;8:58. doi: 10.1186/1471-2199-8-58. PMID: 17605809; PMCID: PMC1931443.

27. Looijenga LH, Hersmus R, Gillis AJ, Pfundt R, Stoop HJ, van Gurp RJ, Veltman J, Beverloo HB, van Drunen E, van Kessel AG, Pera RR, Schneider DT, Summersgill B, Shipley J, McIntyre A, van der Spek P, Schoenmakers E, Oosterhuis JW: Genomic and expression profiling of human spermatocytic seminomas: primary spermatocyte as tumorigenic precursor and DMRT1 as candidate chromosome 9 gene. Cancer Res 2006, 66(1):290-302.

28. Danielsson F, Skogs M, Huss M, Rexhepaj E, O’Hurley G, Klevebring D, Ponten F, Gad AK, Uhlen M, Lundberg E. Majority of differentially expressed genes are down-regulated during malignant transformation in a four-stage model. Proc Natl AcadSci U S A. 2013 Apr 23;110(17):6853-8. doi: 10.1073/pnas.1216436110. Epub 2013 Apr 8. Erratum in: Proc Natl AcadSci U S A. 2015 Dec 22;112(51):E7158. PMID: 23569271; PMCID: PMC3637701.

29. Li WC, Yu WY, Quinlan JM, Burke ZD, Tosh D. The molecular basis of transdifferentiation. J Cell Mol Med. 2005 Jul-Sep;9(3):569-82. doi: 10.1111/j.1582-4934.2005.tb00489.x. PMID: 16202206; PMCID: PMC6741337.

30. Dong $\mathrm{Z}$, Lei Q, Yang $\mathrm{R}$, et al. Inhibition of neurotensin receptor 1 induces intrinsic apoptosis via let-7a-3p/Bcl-w axis in glioblastoma. Br J Cancer. 2017;116(12):1572-1584. doi:10.1038/bjc.2017.126

31. Kontovounisios C, Qiu S, Rasheed S, Darzi A, Tekkis P. The role of neurotensin as a novel biomarker in the endoscopic screening of high-risk population for developing colorectal neoplasia. Updates Surg. 2017 Sep;69(3):397-402. doi: 10.1007/s13304-017-0464-6. Epub 2017 May 30. PMID: 28560510; PMCID: PMC5591352.

32. Brun P, Mastrotto C, Beggiao E, Stefani A, Barzon L, Sturniolo GC, Palu G, Castagliuolo I. Neuropeptide neurotensin stimulates intestinal wound healing following chronic intestinal inflammation. Am J PhysiolGastrointest Liver Physiol. 2005 Apr;288(4):G621-9. doi: 10.1152/ajpgi.00140.2004. PMID: 15764810 .

33. Hansen LA, Sigman CC, Andreola F, Ross SA, Kelloff GJ, De Luca LM. Retinoids in chemoprevention and differentiation therapy. Carcinogenesis. 2000 Jul;21(7):1271-9. PMID: 10874003.

34. Schissel SL, Dunsmore SE, Liu X, Shine RW, Perrella MA, Layne MD. Aortic carboxypeptidase-like protein is expressed in fibrotic human lung and its absence protects against bleomycin-induced lung fibrosis. Am J Pathol. 2009 Mar;174(3):818-28. doi: 10.2353/ajpath.2009.080856. Epub 2009 Jan 29. 
PMID: 19179605; PMCID: PMC2665743.

35. Layne MD, Yet SF, Maemura K, Hsieh CM, Bernfield M, Perrella MA, Lee ME. Impaired abdominal wall development and deficient wound healing in mice lacking aortic carboxypeptidase-like protein. Mol Cell Biol. 2001 Aug;21(15):5256-61. doi: 10.1128/MCB.21.15.5256-5261.2001. PMID: 11438679; PMCID: PMC87249.

36. Nuylan M, Kawano T, Inazawa J, Inoue J. Down-regulation of LAPTM5 in human cancer cells. Oncotarget. 2016 May 10;7(19):28320-8. doi: 10.18632/oncotarget.8614. PMID: 27058622; PMCID: PMC5053729.

37. Niu G, Yang Y, Ren J, Song T, Hu Z, Chen L, Hong R, Xia J, Ke C, Wang X. Overexpression of CPXM2 predicts an unfavorable prognosis and promotes the proliferation and migration of gastric cancer. Oncol Rep. 2019 Oct;42(4):1283-1294. doi: 10.3892/or.2019.7254. Epub 2019 Jul 30. PMID: 31364750; PMCID: PMC6718098.

38. Stevens WW, Staudacher AG, Hulse KE, Carter RG, Winter DR, Kato A, Suh L, Norton JE, Huang JH, Peters AT, Grammer LC, Price CPE, Conley DB, Shintani-Smith S, Tan BK, Welch KC, Kern RC, Schleimer RP. Activation of the 15-lipoxygenase pathway in aspirin-exacerbated respiratory disease. J Allergy Clin Immunol. 2020 May 1:S0091-6749(20)30627-8. doi: 10.1016/j.jaci.2020.04.031. Epub ahead of print. PMID: 32371071.

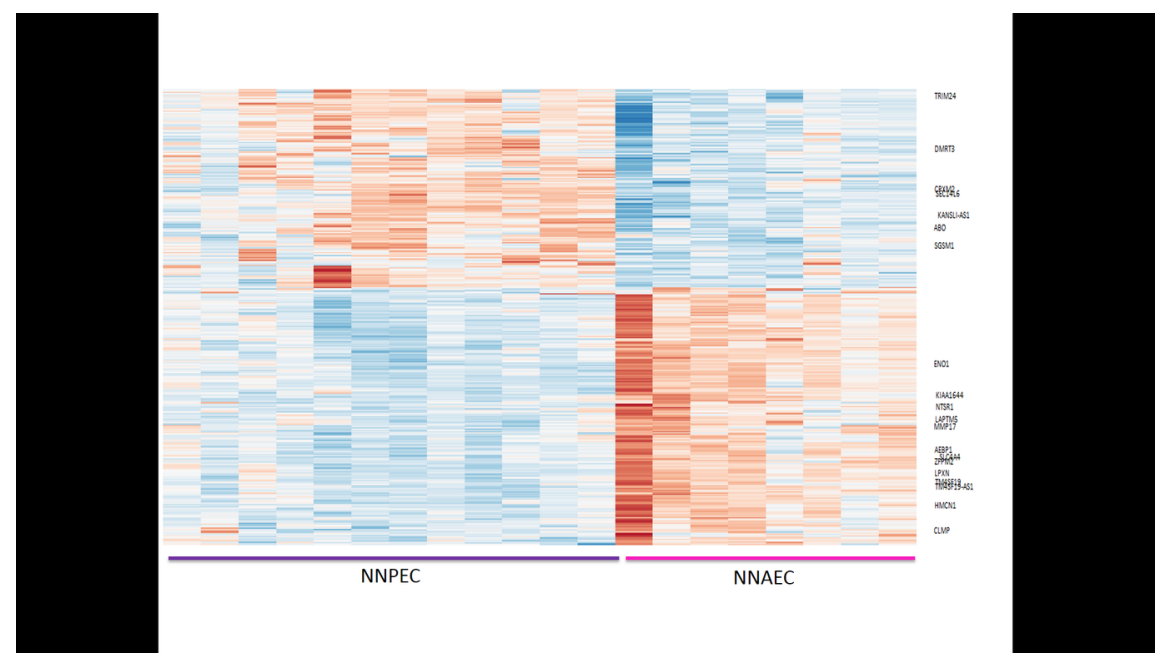

Figure 1: Heatmap showing differential expression of N-ERD nasal polyp epithelial cell (NNPEC) versus N-ERD non affected epithelial cell (NNAEC) 


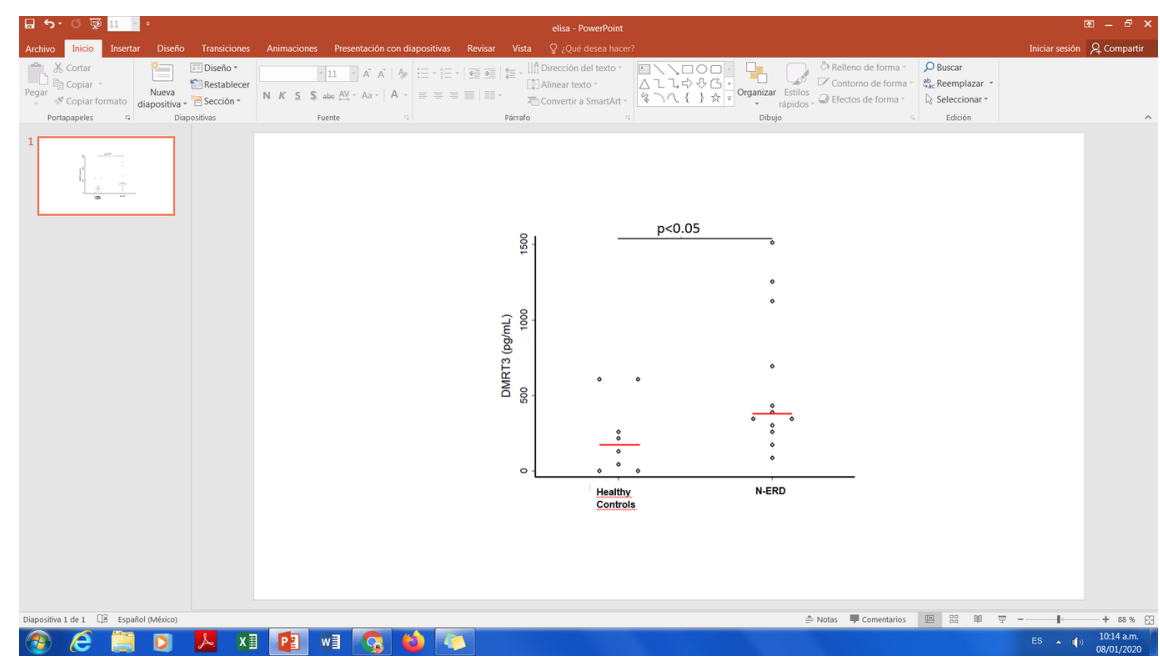

Figure 2: Dot plot showing significant difference in DMRT3 levels in nasal aspirates of N-ERD vs controls.
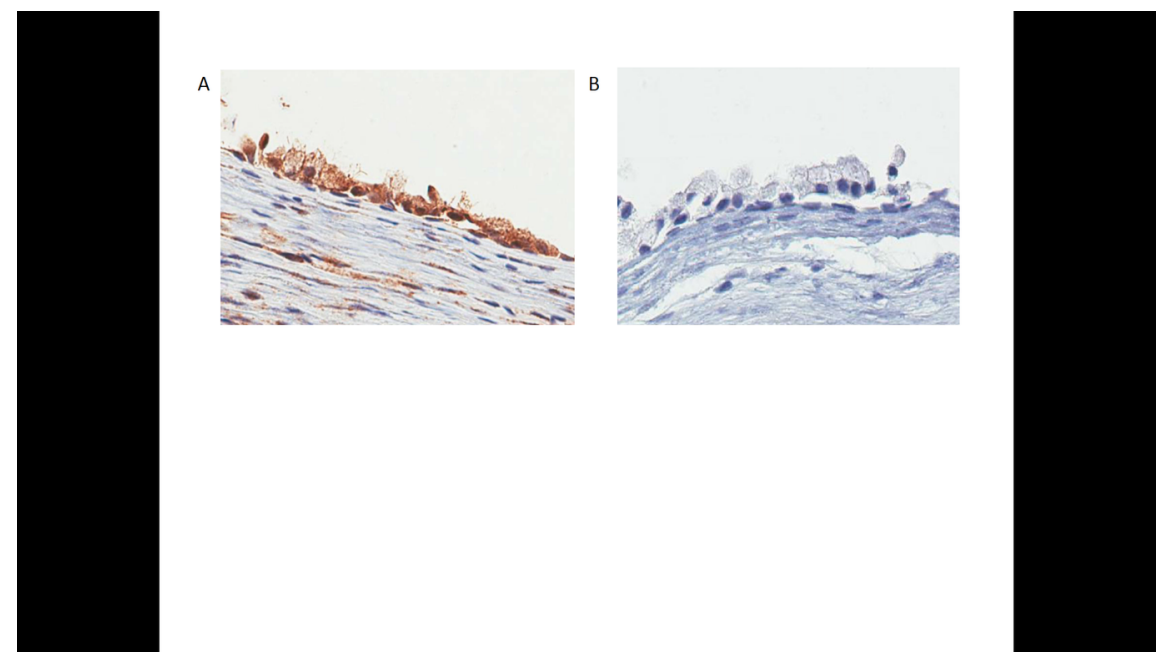

Figure 3: (A) Immunohistochemistry was used to predominantly localize DMRT3 in airway epithelia of nasal polyps from N-ERD patients. (B) Negative control for DMRT3

\begin{tabular}{lll}
\hline Table 1 Clinical Characteristics of Subjects & Table 1 Clinical Characteristics of Subjects & Table 1 Clinical Characteristics \\
\hline & Group A N-ERD & Group B N-ERD \\
No. of patients & 12 & 12 \\
Sex f/m & $9 / 3$ & $9 / 3$ \\
Age (y), median (range) & $54(41-55)$ & $43(33-51)$ \\
FEV1 (\% predicted), median (range) & $87(86-89)$ & $94(90-97)$ \\
Eosinophils (cells/mm $\left.{ }^{3}\right)$, median (range) & $250(150-600)$ & $300(300-400)$ \\
IgE (UI/L) median (range) & $67.3(57-285)$ & $125(90-195)$ \\
Systemic steroids & Dexamethasone 8 mg single dose & None \\
High Grade of Nasal Polyps* n (\%) & $12(100 \%)$ & $7(58 \%)$ \\
Severe Asthma** $\mathrm{n}(\%)$ & $8(66 \%)$ & $10(83 \%)$ \\
\hline
\end{tabular}


* Nasal polyps grade 3 or 4 (Meltzer's grading system) **Asthma that requires high medication to get control N.A., not applicable

Table 2: Differentially expressed genes with log 2-fold change.

\begin{tabular}{lll}
\hline Rownames(resOrdered) $[1: 20]$ & log2Fold change & p value adjusted \\
\hline AEBP1 & $\mathbf{- 2 . 7 0 7 3 6 2 7}$ & $\mathbf{2 . 0 2 8 2 1 9 e - 0 6}$ \\
MMP17 & $\mathbf{1 . 7 7 1 8 3 1 6}$ & $\mathbf{2 . 0 7 6 1 1 5 e - 0 6}$ \\
CPXM2 & $\mathbf{2 . 4 6 5 2 5 3 1}$ & $\mathbf{2 . 0 7 6 1 1 5 e - 0 6}$ \\
CLMP & $\mathbf{- 1 . 9 8 1 7 6 9 4}$ & $\mathbf{2 . 0 7 6 1 1 5 e - 0 6}$ \\
NTRS1 & $\mathbf{- 2 . 4 1 2 5 0 4 6}$ & $\mathbf{3 . 6 8 5 0 8 7 e - 0 5}$ \\
DMRT3 & $\mathbf{2 . 3 5 2 8 2 5 9}$ & $\mathbf{5 . 2 5 1 8 1 5 e - 0 5}$ \\
TM4SF19-AS1 & $\mathbf{- 2 . 1 9 2 6 3 2 0}$ & $\mathbf{6 . 3 1 0 9 8 2 e - 0 5}$ \\
ABO & $\mathbf{1 . 5 8 0 9 8 1 7}$ & $\mathbf{6 . 7 3 3 6 5 1 e - 0 5}$ \\
KIAA1644 & $\mathbf{- 2 . 3 4 7 0 0 8 2}$ & $\mathbf{9 . 3 8 6 2 3 8 e - 0 5}$ \\
HMCN1 & $\mathbf{- 2 . 2 7 2 6 4 6 9}$ & $\mathbf{1 . 1 5 8 0 0 4 e - 0 4}$ \\
LAPTM5 & $\mathbf{- 2 . 2 2 7 1 4 2 4}$ & $\mathbf{1 . 6 1 6 7 5 1 e - 0 4}$ \\
ZFPM2 & $\mathbf{- 2 . 2 2 2 8 2 9 8}$ & $\mathbf{3 . 0 8 1 2 4 4 e - 0 4}$ \\
SGSM1 & $\mathbf{2 . 0 3 6 6 4 6 5}$ & $\mathbf{6 . 5 5 5 1 3 1 e - 0 4}$ \\
LPXN & $\mathbf{- 1 . 9 9 5 4 1 3 8}$ & $\mathbf{9 . 2 5 8 3 3 5 e - 0 4}$ \\
ENO1 & $\mathbf{- 0 . 8 0 2 6 3 6 7}$ & $\mathbf{9 . 7 7 4 8 0 6 e - 0 4}$ \\
TM4SF19 & $\mathbf{- 2 . 0 2 8 4 7 4 2}$ & $\mathbf{1 . 1 7 4 3 0 4 e - 0 3}$ \\
SLC4A4 & $\mathbf{- 2 . 0 2 6 0 9 2 4}$ & $\mathbf{1 . 5 7 8 0 3 4 e - 0 3}$ \\
TRIM24 & $\mathbf{0 . 6 1 3 4 8 6 8}$ & $\mathbf{1 . 5 7 8 0 3 4 e - 0 3}$ \\
KANSL1-AS1 & $\mathbf{1 . 6 2 1 5 6 0 3}$ & $\mathbf{2 . 1 6 2 7 7 7 e - 0 3}$ \\
SEC14L6 & $\mathbf{1 . 9 5 4 2 7 5 0}$ & $\mathbf{2 . 1 7 5 9 7 2 e - 0 3}$ \\
\hline
\end{tabular}

Table 3: Pathways enriched between NPEC versus NNAE using pathview

\begin{tabular}{lll}
\hline id & pathway & $\mathrm{p}$ value \\
\hline hsa00830 & Retinol metabolism & 0.006416688 \\
hsa00140 & Steroid hormone biosynthesis & 0.017377887 \\
hsa00120 & Primary bile acid biosynthesis & 0.017620697 \\
hsa00360 & Phenylalanine metabolism & 0.04440799 \\
\hline
\end{tabular}

Table 4: Pathways enriched between NPEC versus NNAE using GO enrichment analysis

\begin{tabular}{lll}
\hline id & pathway & $\mathrm{p}$ value \\
\hline GO:0007009 & Plasma membrane organization & 0.006603899 \\
GO:0016236 & Macroautophagy & 0.009654063 \\
GO:0006691 & Leukotriene metabolic process & 0.011124361 \\
GO:0072659 & Protein localization to plasma & 0.012383519 \\
& membrane & \\
GO:0042737 & Drug catabolic process & 0.015426792 \\
GO:0007158 & Neuron cell-cell adhesion & 0.017589775 \\
GO:0009812 & Flavonoid metabolic process & 0.022826975 \\
GO:0000045 & Autophagic vacuole assembly & 0.023120577 \\
GO:0048713 & Regulation of oligodendrocyte & 0.023746775 \\
& differentiation &
\end{tabular}




\begin{tabular}{lll}
\hline id & pathway & $\mathrm{p}$ value \\
\hline GO:0000042 & Protein targeting to Golgi & 0.024045644 \\
GO:0000301 & Retrograde transport, vesicle & 0.024312565 \\
& recycling within Golgi & \\
\hline
\end{tabular}

